\title{
Androgens and COVID-19: exploring the role of testosterone replacement therapy
}

\author{
Nahid Punjani iD $^{1 凶}$ and Ryan Flannigan (iD ${ }^{1,2}$ \\ (c) The Author(s), under exclusive licence to Springer Nature Limited 2021
}

IJIR: Your Sexual Medicine Journal (2022) 34:649-651; https://doi.org/10.1038/s41443-021-00524-6

\section{INTRODUCTION}

A severe atypical pneumonia was discovered in Wuhan, China in December 2019 which was later determined to be caused by SARS-CoV-2 (severe acute respiratory syndrome coronavirus 2) [1]. To date, nearly over 175 million cases of coronavirus disease 2019 (COVID-19) have been reported worldwide, with over 3.8 million deaths, and over 2.3 billion vaccines administered [2]. Since its discovery, an abundance of studies have been conducted to better understand the complex pathophysiology of the disease, its consequences to the human body and epidemiologic trends to elucidate groups that are at greatest risk. A multitude of evidence was available early in the pandemic highlighting a clear sexdifference with greater burdens of disease in men including worse outcomes (both hospitalization and death) even when considering known COVID-19 risk factors, such as age [3]. In an effort to better understand this trend many studies have proposed hypotheses to explain these differences, one of which has included the possible role of androgens [4, 5]. However, to date the relationship between testosterone and COVID-19 remains unclear and somewhat controversial. Here we review COVID-19 pathophysiology, explore some hypotheses for the observed sex differences, interrogate the role of androgen states in COVID-19 and highlight the evidence of testosterone replacement therapy (TRT).

\section{COVID-19 PATHOGENESIS}

COVID-19 is a single-stranded RNA virus transmitted through respiratory droplets with an incubation period of 2-14 days. Structurally, COVID-19 consists of an inner nucleoprotein encapsulated by an envelope which on its surface has spike proteins necessary for viral infectivity [6]. The spike proteins engage with the host via direct binding of the angiotensin-converting enzyme 2 (ACE2) receptor, a key component of the renin-angiotensinaldosterone system necessary for human fluid and blood pressure regulation [6]. Spike proteins are further primed through engagement of the transmembrane protease serine 2 (TMPRSS2) receptor [6]. Both of these have been discovered as critical for both infection and viral entry emphasizing their importance in the disease process. While ACE is well known in the respiratory system, secondary to its production in the lungs following stimulation of renin produced by the kidney, receptors for ACE2 and TMPRSS2 have actually been identified in multiple diffuse organ systems throughout the body. Specific to males, receptors have been identified in both the prostate and testis, as well as specifically Sertoli cells, Leydig cells and spermatogonia [7].

\section{SEX DIFFERENCE AND COVID-19}

Numerous reports have attempted to understand the sexdifference observed in COVID-19, whether secondary to an innate sex-variable physiology or acquired from lifestyle and behavior $[3,8]$. Initially, it was hypothesized that the presence of receptors on male-specific tissue was contributory to some of the observed differences, including some clinical presentations such as orchitis. From a lifestyle perspective it was hypothesized that the presence of additional comorbidities in men and/or higher proportions of high-risk behaviors such as smoking were responsible, but this independently has been an insufficient explanation [3]. Alternatively, since ACE2 is an X-linked gene, it has been suggested that differential $X$ chromosome inactivation may be in part responsible for the differences [8]. Furthermore, since TMPRSS2 is an androgen-regulated gene, as discovered through the ERG: TMPRSS2 fusion gene in prostate cancer, this has been suggested to possibly provide insight into a potential hormonal dependent explanation for the sex difference [8]. Finally, there is evidence to suggest differential immune responses based on sex that is driven by hormonal impacts of progesterone and estrogen on the immune system, a finding which is supported by increased rates of COVID-19 in post-menopausal women [8]. However, to date these hypotheses have not been entirely sufficient to explain the observed differences.

\section{COVID-19 AND MALE REPRODUCTIVE HORMONES}

Given these hypotheses, it seemed plausible that a hormonal difference, such as differences in androgen levels, play a pivotal role in the COVID-19 infection. However, to date, the evidence has been mixed, with some data supporting the concept that high androgen states confer worsened disease and other reports suggesting that low testosterone states are associated with more severe outcomes. Overall this divide has illustrated that perhaps there is a complex interplay with androgens, and specifically testosterone, in COVID-19 and suggests some persistent caution when manipulating these hormones in an effort to mitigate the COVID-19 disease course.

\footnotetext{
${ }^{1}$ Department of Urology, Weill Cornell Medicine, New York, NY, USA. ${ }^{2}$ Department of Urologic Sciences, University of British Columbia, Vancouver, BC, Canada.

凶email: nap4001@med.cornell.edu
}

Received: 14 June 2021 Revised: 16 December 2021 Accepted: 20 December 2021

Published online: 30 January 2022 
One of the first studies suggesting a hormonal difference in COVID-19 outcomes occurred in a group of men with prostate cancer on androgen depravation therapy (ADT). This study examined a total of 4532 men from 68 hospitals in the Italian region of Veneto as of April 1, 2020. They found that men with prostate cancer who were not on ADT had significantly higher odds of disease positivity as well as both mild and severe disease [4]. These data implied that a hormone deficient, effectively hypogonadal, state offered protection against COVID-19 and its subsequent outcomes. As an extension to this, other hormone modulating therapies could also be considered such as five-alpha reductase inhibitors, which block the conversion of testosterone to dihydrotestosterone, or medications such as spironolactone, a steroidal anti-androgen, to also offer the same protection [9]. In the five-alpha reductase inhibitor study, they examined 1432 COVID-19 patients from two high volume centers in Italy from March $1^{\text {st }}$ to April $24^{\text {th }} 2020$ specifically targeting men who had received long-term treatment. The evidence for these alternative hormone-modulating therapies is less impressive but does provide some support that hormone manipulation may moderate the effects of COVID-19.

In general, it has been challenging to study acute serum hormone levels at the time of COVID-19 illness, given the known variations that occur with acute illness, the known physiological variations, and the lack of pre-COVID comparator hormone data in many studies [10]. Nonetheless, in opposition to the protective hypogonadal state, various studies have demonstrated that low testosterone states are associated with worse disease. One of the first reports to present hormone data during COVID-19 infection was a retrospective series which demonstrated that men with SARS-CoV-2 infection, compared to age-matched healthy men, had comparable levels of testosterone but had higher luteinizing hormone (LH) and prolactin, as well as a lower testosterone to $\mathrm{LH}$ ratio [11]. The authors suggest that this state may be representative of compensated hypogonadism, with elevated LH representing the bodies attempt to maintain testosterone levels. Another study demonstrated that in a cohort of men admitted to hospital, almost $90 \%$ of these men had evidence of acquired hypogonadism and of these, $85 \%$ of cases were secondary [12]. This was not surprising as low testosterone levels have also been associated with severe illnesses and respiratory failure $[13,14]$. In their report, they also found that lower testosterone levels were associated with worse outcomes, the opposite of the previous ADT study [12]. More recently, a prospective study was conducted which assessed serum hormone levels at multiple time points including at the time of presentation, and up to four subsequent time points (up to 28 days) post admission if they were hospitalized and data were available [5]. They compared 24 men who never had severe COVID-19 of which no patient was hospitalized beyond 7 days, to 31 men who had severe COVID-19 at presentation, and 35 men who developed severe COVID-19. They found that men with severe COVID had significantly lower testosterone levels up to 7 days post presentation as well as at baseline.

An understanding of the role of testosterone in other disease processes may also be helpful to understand and support the relationship between hypogonadal states and poor COVID-19 outcomes. Since testosterone production declines in men after age 30 , at a rate of $1-2 \%$ per year, there is a resultant greater rate of hypogonadism in elderly men [15]. This supports the currently observed increase in worse COVID-19 outcomes as well as death in men of increasing age [3]. While a similar trend was also observed for aging women, this may be secondary to menopause and a loss of immune protection from progesterone and estrogen [16]. There are also numerous associations between hypogonadism and various medical comorbidities, some of which are also risk factors for COVID-19, including diabetes, sleep apnea and metabolic syndrome [5]. Men with hypogonadism have also been shown to have worsened respiratory outcomes, such as in chronic obstructive pulmonary disorder, further potentiating the worse COVID-19 disease course in men with low testosterone [17].

There is however an important caveat to consider here since there is data to support hypogonadal men secondary to ADT with protective outcomes, as well as opposing data to suggest worse outcomes in otherwise hypogonadal men. Taken together, this indicates that these two groups of individuals are inherently different and not comparable simply because of a similar testosterone level, and perhaps there is an underlying physiologic difference in these individuals that requires further investigation. Furthermore, even amongst those who are hypogonadal without the use of ADT, is it that the lower testosterone is responsible for worse outcomes or does severe disease result in a more prominent decline in testosterone? [18] Answers to these questions are critical when considering the role of androgens in COVID-19 as well as considering testosterone replacement therapy (TRT) in COVID-19.

\section{TESTOSTERONE REPLACEMENT THERAPY AND COVID-19}

TRT aims to improve men with documented testosterone deficiency $(<300 \mathrm{ng} / \mathrm{dl})$ and associated signs and/or symptoms of testosterone deficiency including reduced energy, loss of muscle mass, diminished libido, altered mood, abnormal bone mass and poorly controlled sugars $[19,20]$. TRT may be provided in multiple forms including oral, buccal, nasal, gels, pellets, and intramuscular injections with variable benefits and efficacy rates $[19,20]$. Testosterone is however not benign, including reported increased risks of thromboembolic events, and is contraindicated in both prostate and breast cancer, polycythemia, severe heart failure, severe urinary symptoms, obstructive sleep apnea, and those desiring fertility as it inhibits spermatogenesis [19].

So, if there is an androgen-dependent effect on COVID-19, it seems plausible that hormone manipulation, in the form of TRT, could provide a possible solution to mitigate disease severity. To date, while there have been other hormone manipulation studies (i.e., progesterone) demonstrating symptomatic improvement, limited data exists for TRT [21]. That said, testosterone administration in hypogonadal men may have a logical role in the COVID19 pathophysiology as men with testosterone deficiency tend to have elevated levels of pro-inflammatory cytokines including IL-6, IL-1, and TNF-alpha [22]. In these men, the administration of testosterone supplementation is thought to blunt this inflammatory response, and therefore reduce the possible cytokine storm which arises from COVID-19, thus alleviating some of the severe disease outcomes secondary to respiratory compromise.

Furthermore, as previously mentioned, testosterone has a protective role in some systemic conditions including bone health and diabetes [20]. Since testosterone in deficient patients has been shown to reduce hospitalization, all-cause mortality and prevention of diabetes, it provides overall health benefits [23]. As an extension, since comorbidities such as diabetes are a wellknown risk factor for COVID-19, strategies to prevent this and other hypogonadism related conditions may theoretically diminish hospitalization and/or death rates [1].

To date there are limited cohort studies of TRT and COVID-19. One recent retrospective study examined 32 men on TRT who were propensity-score-matched to 63 men not on TRT, in an effort to have comparable populations on factors other than TRT usage [24]. In their report they interestingly found no difference in outcomes including hospital admission, intensive care stay, use of mechanical ventilators, and death. This suggests that hormonal correction of men into a eugonadal state may be removing the harmful effects that have been observed from hypogonadism. Importantly, the authors also found no difference for thromboembolic events, a known concern for TRT administration.

This begs the question, is it safe to administer testosterone to men with hypogonadism and COVID-19? To date, the evidence is 
insufficient to determine if there is a therapeutic benefit of TRT initiation, and therefore this should be approached with caution. For men previously on TRT with new COVID-19 infection, this group likely warrants closer attention. Given the side effects of TRT including impacts to hematocrit and thyroid hormone levels, as well as the systematic impacts of COVID-19, these patients will likely require close follow-up [25]. Furthermore, given the unknown risk of COVID-19 long term, TRT and non-TRT patients may require closer and more rigorous follow-up than currently suggested by standard guidelines, until more definitive data becomes available.

\section{THE NEED FOR FURTHER STUDIES}

To date, the long-term impact of testosterone on COVID-19 remains to be clearly elucidated, and from the available data, testosterone may be still be acting as a double-edged sword. While there have been some reports suggesting that a low testosterone state is protective, there is increasingly strong evidence suggesting a negative impact of hypogonadism and COVID-19. This however has been insufficient to independently explain the observed sex difference in COVID-19. Overall, the mechanisms are likely multidimensional and interdependent on a wide variety of other factors. Longitudinal studies are needed to further understand the long-term impact of hormones in the context of COVID-19, and prospective studies are required to provide definitive evidence of the role of TRT both as a treatment, and the optimal management of patients already on TRT.

\section{REFERENCES}

1. Singh A, Shaikh A, Singh R, Singh AK. COVID-19: from bench to bed side. Diabetes Metab Syndr. 2020;14:277-81.

2. Medicine JHU COVID-19 Dashboard by the Center for Systems Science and Engineering (CSSE). 2021. https://coronavirus.jhu.edu/map.html.

3. Punjani N, Ha A, Captuo J, Wang V, Wiechmann L, Chiasson MA, et al. Outcome disparities amongst men and women with COVID-19 (Coronavirus Disease 2019): an analysis of the New York City Population Cohort. J Drugs Dermatol. 2020;19:960-7.

4. Montopoli M, Zumerle S, Vettor R, Rugge M, Zorzi M, Catapano CV, et al. Androgen-deprivation therapies for prostate cancer and risk of infection by SARSCoV-2: a population-based study ( $N=4532)$. Ann Oncol. 2020;31:1040-5.

5. Dhindsa S, Zhang N, McPhaul MJ, Wu Z, Ghoshal AK, Erlich EC, et al. Association of circulating sex hormones with inflammation and disease severity in patients with COVID-19. JAMA Netw Open. 2021;4:e2111398.

6. Hoffmann M, Kleine-Weber H, Schroeder S, Kruger N, Herrler T, Erichsen S, et al. SARS-CoV-2 cell entry depends on ACE2 and TMPRSS2 and is blocked by a clinically proven protease inhibitor. Cell. 2020;181:271-80. e8

7. Sungnak W, Huang $N$, Becavin $C$, Berg $M$, Queen R, Litvinukova $M$, et al. SARSCoV-2 entry factors are highly expressed in nasal epithelial cells together with innate immune genes. Nat Med. 2020;26:681-7.

8. Patel DP, Punjani N, Guo J, Alukal JP, Li PS, Hotaling JM. The impact of SARS-CoV2 and COVID-19 on male reproduction and men's health. Fertil Steril. 2021;115:813-23.

9. Lazzeri M, Duga S, Azzolini E, Fasulo V, Buffi N, Saita A, et al. Impact of chronic exposure to 5-alpha reductase inhibitors on the risk of hospitalization for COVID19: a case-control study in male population from two COVID-19 regional centers of Lombardy (Italy). Minerva Urol Nefrol. 2021. https://pubmed.ncbi.nlm.nih.gov/ 33439572/ (Online ahead of print).

10. Schroeder M, Tuku B, Jarczak D, Nierhaus A, Bai T, Jacobsen $H$, et al. The majority of male patients with COVID-19 present low testosterone levels on admission to Intensive Care in Hamburg, Germany: a retrospective cohort study. medrxiv. 2020. Online - https://www.medrxiv.org/content/10.1101/2020.05.07.20073817v1.full. pdf + html.
11. Ma L, Xie W, Li D, Shi L, Mao Y, Xiong Y, et al. Effect of SARS-CoV-2 infection upon male gonadal function: a single center-based study. medRxiv. 2020. Online https://doi.org/10.1101/2020.03.21.20037267.

12. Salonia A, Pontillo M, Capogrosso P, Gregori S, Tassara M, Boeri L, et al. Severely low testosterone in males with COVID-19: a case-control study. Andrology. 2021;9:1043-52.

13. Almoosa KF, Gupta A, Pedroza C, Watts NB. Low testosterone levels are frequent in patients with acute respiratory failure and are associated with poor outcomes. Endocr Pr. 2014;20:1057-63.

14. Christeff N, Benassayag C, Carli-Vielle C, Carli A, Nunez EA. Elevated oestrogen and reduced testosterone levels in the serum of male septic shock patients. J Steroid Biochem. 1988;29:435-40.

15. Kaufman JM, Vermeulen A. The decline of androgen levels in elderly men and its clinical and therapeutic implications. Endocr Rev. 2005;26:833-76.

16. Ghosh M, Rodriguez-Garcia M, Wira CR. The immune system in menopause: pros and cons of hormone therapy. J Steroid Biochem Mol Biol. 2014;142:171-5.

17. Baillargeon J, Urban RJ, Zhang W, Zaiden MF, Javed Z, Sheffield-Moore M, et al. Testosterone replacement therapy and hospitalization rates in men with COPD. Chron Respir Dis. 2019;16:1479972318793004.

18. Spratt DI, Bigos ST, Beitins I, Cox P, Longcope C, Orav J. Both hyper- and hypogonadotropic hypogonadism occur transiently in acute illness: bio- and immunoactive gonadotropins. J Clin Endocrinol Metab. 1992;75:1562-70.

19. Barbonetti A, D'Andrea S, Francavilla S. Testosterone replacement therapy Andrology. 2020;8:1551-66.

20. Mulhall JP, Trost LW, Brannigan RE, Kurtz EG, Redmon JB, Chiles KA, et al. Evaluation and management of testosterone deficiency: AUA guideline. J Urol. 2018;200:423-32.

21. Ghandehari S, Matusov Y, Pepkowitz S, Stein D, Kaderi T, Narayanan D, et al. Progesterone in addition to standard of care vs standard of care alone in the treatment of men hospitalized with moderate to severe COVID-19: a randomized, controlled pilot trial. Chest. 2021;160:74-84.

22. Mohamad NV, Wong SK, Wan Hasan WN, Jolly JJ, Nur-Farhana MF, Ima-Nirwana S, et al. The relationship between circulating testosterone and inflammatory cytokines in men. Aging Male. 2019;22:129-40.

23. Hackett G, Kirby M. Testosterone deficiency in men infected with COVID-19. Trends Urol Mens Health. 2020;11:7-10.

24. Rambhatla A, Bronkema CJ, Corsi N, Keeley J, Sood A, Affas Z, et al. COVID-19 infection in men on testosterone replacement therapy. J Sex Med. 2021;18:215-8.

25. Hussain AN, Hussain F, Hashmi SK. Role of testosterone in COVID-19 patients - a double-edged sword? Med Hypotheses. 2020;144:110287.

\section{AUTHOR CONTRIBUTIONS}

Conception, Design and Interpretation - RF and NP. Drafting and Editing - RF and NP.

\section{FUNDING}

NP is supported in part by the Frederick J. and Theresa Dow Wallace Fund of the New York Community Trust.

\section{COMPETING INTERESTS}

The authors declare no competing interests.

\section{ADDITIONAL INFORMATION}

Correspondence and requests for materials should be addressed to Nahid Punjani.

Reprints and permission information is available at http://www.nature.com/ reprints

Publisher's note Springer Nature remains neutral with regard to jurisdictional claims in published maps and institutional affiliations. 